\title{
Development of Remote Wireless Communication System of Smart
}

\section{Home}

\author{
Ke Yuan ${ }^{1}$ \\ ${ }^{1}$ Nanchang Institute of Science and Technology, Nanchang, Jiangxi, 330108 \\ 346591653@163.com
}

\section{KEYWORDS: Wireless; Communication System; Smart Home}

\begin{abstract}
Traditional smart home system is mainly used to form a wired manner, such as RS-485/X10/Lonworks/CAN and the like. Add or delete a wired network equipment will involve changes to the line, and large projects affect the interior decoration of beautiful, poor scalability of its systems, installation, commissioning and maintenance costs are high. To solve these problems, the present situation of the development of wireless network communication technology, this paper will examine system design smart home wireless network systems, network infrastructure, the main controller (intelligent gateway), the relay device (radio repeater) and a terminal-based nodes (wireless lighting controller) design.
\end{abstract}

\section{Introduction}

Connotation smart home system is "in a residential home with a personalized, the pluralistic network information, control, and automation features diversification of energy saving and environmental protection, integrated into the family of intelligent integrated information management and automated monitoring platform." With the development of these years, smart home has begun to spread in the country, there are some real estate developers were smart home Property standardized configuration to attract customers to purchase. They want to be able to control external devices via smart home system at home, such as air conditioners, water heaters, home can enjoy hot and cold moderate indoor temperature, smart water heater has been put away hot water; at home can be a key to control all appliances, intelligent home system so by household habits adjusted to the most comfortable living environment. For the sales of real estate developers characteristics - mainly rough delivery room floor, the owners can not predict the decor, the line can not be embedded smart home network, you can not pre-installed intelligent home equipment, decoration materials can not predict the impact of a control signal, etc. etc., but the contract he promised to send the whole house smart home, which seems to be contradictory, relying on the traditional smart home system has been unable to solve.

\section{The Choice of the Smart Home System Control and Wireless Sensor Networks}

The ultimate goal of smart home system is to give users a more convenient and secure life, so a good smart home system should have a low cost, easy to use, good flexibility, high stability, security and other advantages. Select the smart home system control chip and wireless sensor networks within the home network will have an important impact on the cost of smart home system, stability, security, flexibility and maneuverability. Home network build wireless sensor networks is 
an important part of the smart home system. Home control commands transmitted within the network reliability, security, smart home rate is the main basis for selection of wireless sensor networks. According to the actual needs of the smart home system, smart home system in the home network using a wireless sensor network to pass major home control command, based on the satisfaction of basic functional requirements, but also within the home network node of wireless sensor networks have a smaller volume and strong flexibility. To sum up, smart home system for wireless sensor network node should have a low-cost, low-rate, low power consumption, small size and high flexibility. Construction of the network in the traditional home of smart home system in the use of wired connections, wired connections with high costs, complicated wiring, easy maintenance, and other defects affecting indoor appearance. Therefore, the wireless network technology for smart home internal network has become a trend, here are some commonly used short-range wireless network technology: Bluetooth technology, infrared technology, Wi-Fi technology, ZigBee technology-based Basic-RF two-way communications technology.

\section{Smart Home System Design}

Currently, intelligent home systems are typically used in high-end hotels, residential and large-scale buildings. The current smart home system family of ordinary people did not get a wide range of promotion. In addition to the current user acceptance of smart home is still relatively low outside the main there are three reasons, first wiring complexity is not conducive to the completion of construction in the residential arrangements; secondly, the lack of smart home system can be customized by the user personalized service; and finally, intelligent home systems generally costly, hindering large-scale promotion in the smart home of the people. (1), the traditional smart home wiring system complex issue, this paper within the home network communication mode tree-based wireless sensor networks. For household appliances control command data, their network bandwidth requirements low, and wireless sensor networks based on Texas Instruments CC2530 chip Basic-RF communication protocol is a low speed, low power, low cost, high stability of communication can fully meet the home control command stable transmission within the home network. In addition, the wireless sensor network nodes of the smart home system the flexibility to add or remove the user's needs, so the use of the smart home system, smart home system can solve the traditional routing complex issues.

For intelligent home systems lack the personalized service problem, in this smart home system to do some improvements. In this paper, the use of smart home system, users have the flexibility to easily select or remove access intelligent home network electrical equipment. In addition, home interior, the user can use the remote control system and say "lights," "Lights," "turn on the TV" and other simple voice command can make the system complete the corresponding control function; the same time, users can also comfortably lying in bed, using a mobile phone or tablet can change the room light and sound effects; smart home remote control function allows users though thousands of miles away, as long as through the Internet or mobile phone text messages to learn the family information, complete household equipment remotely control. The above control modes can be set by the user like system, so that the smart home system can meet the service requirements of individual users.

Intelligent home control system consists of three parts master module, a wireless sensor network routing nodes and terminal nodes for wireless sensor networks. Remote users can open the master Web server module via the Internet web page (the user has a public IP) or send it via GSM network SMS send control commands to the master module; local user can be directly connected into the system where the network router to send control commands to the control module, you can also turn 
on smart home system client software or use the remote control system complete home control devices. Internal network in the home, the terminal node containing appliance control module, between the master module and the terminal node completes transmission of information via wireless sensor networks. In order to ensure stable transmission of information, to join the wireless sensor network routing nodes each room in the home is responsible for data forwarding and network path maintenance.

\section{Smart Home Communication System Design}

Wireless sensor network is an important part of the smart home system, build a stable, efficient and meet the actual needs of a tree smart home wireless sensor network and application-layer algorithm program based on the actual need to select the Texas Instruments CC2530 chip Basic RF wireless communication protocol like wireless sensor networks. Make the application layer (Application) algorithm will build on the Basic RF layer, so that the wireless sensor network nodes form a tree network, each node has automatic detection reconnect feature, users can increase the flexibility of network nodes according to demand deletion, a damaged node does not affect the transmission of information throughout the network, greatly improving the flexibility and stability of the system.

Basic-RF provided by TI company, which includes the IEEE 802.15.4 standard packet transceiver functions but does not use the stack, it is just to make a simple two nodes to communicate, that Basic-RF It contains just a small part of the IEEE 802.15.4 standard only. Its main features are: 1 , is not automatically added to the agreement, it will not automatically scan other network nodes and no lights. 2, there is no protocol stack inside said coordinator, router or end the distinction between the status of the node is equal. 3, there is no automatic retransmission function. Basic RF layer for the two-way radio communication provides a simple protocol, this protocol can send and receive data. Basic-RF CCM-64 also provides authentication and data encryption for secure communication, the user can define in the project file inside SECURITY_CCM for data encryption. As shown in Figure 4.1. In Project-> Option which can be selected by default in this smart home system, no data encryption, so in front of the $\mathrm{X}$ band security_ccm.

Smart home wireless sensor network and application-layer algorithm program to build a stable, efficient and meet the actual needs of the tree in wireless sensor networks based on the actual need to select the Texas Instruments CC2530 chip Basic-RF wireless communication protocol, mainly for low-bandwidth intelligent home network messaging. Wireless sensor network by the coordinator node, routing nodes and end nodes, the coordinator node is the core of wireless sensor networks, home users to send control commands to be sent to the last coordinator node wireless sensor networks. Wireless sensor network routing nodes for data transfer, the actual application, set up a routing node every room in the home to ensure that all home control commands could eventually spread to the terminal node electrical control terminals. After the intelligent home control module users get sent home control command information, additional information on the route information transmitting control module wireless sensor network coordinator node wireless sensor network coordinator node sends to the corresponding routing node and the terminal node according to the path and eventually to send control commands to the corresponding home information terminal equipment. Household equipment terminal receives the control command information sent in response to feedback control after the completion of information, feedback information and control command path the same information, but in the opposite direction. 
Wireless sensor network initialization process: First, the wireless sensor network coordinator node initialization, smart home system to read the total number of routing nodes, and to initialize the appropriate state information array. Open the wireless sensor network routing node, routing nodes will continue to look for the coordinator node and routing node number than their own numbers, if found a connection is established, determine its own parent node sends its own address information to the parent and to complete the routing node initialization. Routing nodes under normal operating conditions, will regularly send to the parent node detection information, such as receive feedback detection is knowing disconnected from the network, then the routing node will restart, re-connect the parent seeking to ensure that the routing node has been automatically It connected to wireless sensor networks. Open the wireless sensor network terminal node, a terminal node will continue to look for the coordinator node and routing node, if found to establish a connection to determine its own parent node sends its own address information to the parent, and complete the initialization of the terminal node. Terminal nodes under normal operating conditions, will regularly send information to the parent node detection, such as detection not receive feedback, you knew disconnected from the network, then the terminal node restart, find the parent node connected to ensure the terminal node has been automatically connected smart home wireless sensor networks.

\section{Conclusion}

The smart home wireless sensor network system can only deliver low-bandwidth data command. The next step may be Wi-Fi signal is added to the home wireless sensor network transfer video, audio, high-bandwidth signals, while the network cameras, security equipment added to the smart home system, make the smart home system to provide users with stronger security and convenience.

\section{REFERENCE:}

[1] Man S, Yang H X, Peng Y, et al. Design of embedded wireless smart home gateway based on ARM9 [J]. Jisuanji Yingyong/ Journal of Computer Applications.2010, 30(9): 2541-2544.

[2] Shahin Farahani. ZigBee Wireless Networks and Transceivers [M]. USA: Newnes,2008:P3

[3] Raghavan R; Gopikrishnan M. Data Structures[M]. LAP Lambert Academic Publishing, 2010: P22. 